\title{
Efeitos de Três Métodos Complementares à Aspiração nas Repercussões Hemodinâmicas e Mecânica Respiratória em Pacientes Sob Ventilação Mecânica Invasiva
}

\section{The effects of three complementary aspiration methods in hemodynamic repercussions and respiratory mechanics of patients in mechanical invasive ventilation}

\author{
Camila Chaves Bezerra ${ }^{1}$, Débora Ximenes de Águila ${ }^{1}$, Framartinho Carlos Silva Araújo ${ }^{2}$, Guilherme Pinheiro Ferreira da Silva ${ }^{3}$ \\ 1 Acadêmica do curso de graduação em Fisioterapia da Universidade de Fortaleza (UNIFOR). 2 Mestre em Saúde da Criança e do Adolescente pela \\ Universidade Estadual do Ceará (UECE), professor do curso de Fisioterapia da Universidade de Fortaleza (UNIFOR). 3 Mestre em Ciências Médicas \\ pela Universidade Federal do Ceará (UFC).
}

\begin{abstract}
Resumo
Introdução: A remoção de secreção das vias aéreas por meio da aspiração auxilia na higiene brônquica, troca gasosa e melhora da mecânica ventilatória. Este estudo tem como objetivo analisar os efeitos de três métodos complementares à aspiração nas repercussões hemodinâmicas e mecânica respiratória em pacientes sob ventilação mecânica invasiva. Métodos: Participaram do estudo 45 pacientes com diagnóstico clínico de insuficiência respiratória internados na Unidade de Terapia Intensiva, divididos aleatoriamente em três grupos compostos por 15 indivíduos cada: Grupo Ressuscitador Manual (RM), Grupo Fração inspirada de Oxigênio (FiO2) e grupo Ventilação Mecânica (VM). As variáveis analisadas consistiram nos parâmetros da mecânica respiratória e cardiorrespiratórios: frequência cardíaca, frequência respiratória, pressão arterial sistólica, pressão arterial diastólica, saturação periférica de oxigênio, pressão de pico, pressão de platô, complacência estática, complacência dinâmica. A coleta de dados ocorreu em quatro momentos distintos: antes da realização do procedimento, imediatamente após, 5 minutos e 30 minutos após o procedimento. Resultados: Os resultados apontaram que houve repercussões hemodinâmicas e na mecânica respiratória nos três grupos analisados. Quando comparado os grupos, encontrou-se diferença significativa na variável frequência respiratória no período 30 minutos pós-intervenção do grupo RM versus o grupo VM ( $p=0,007$ ), variável P. Pico no período 30 minutos pósintervenção nos grupos RM versus FiO2 (p=0,005) e na variável C. Dinâmica no período 30 minutos pós-intervenção nos grupos FiO2 e VM $(\mathrm{p}=0,005)$. Conclusão: Conclui-se que houve repercussões hemodinâmicas e de mecânica respiratória nos três métodos complementares à aspiração, porém todos são considerados aplicáveis.
\end{abstract}

Palavras-chave: Sucção. Mecânica Respiratória. Hemodinâmica.

\begin{abstract}
Introduction: The airway clearance by endotracheal suctioning helps at bronchial hygiene, gas exchange and respiratory mechanics. The aim of this study was to analyze the repercussion of three suction complementary methods at respiratory mechanics and hemodynamics in invasive mechanically ventilated patients. Methods: Participated 45 patients with diagnosis of respiratory failure that was in Intensive Care Unit, they were randomly divided in three groups, with 15 patients each one: Manual Ressuscitator Group (RM), Fraction of Inspired Oxygen Group (FiO2) and Mechanically Ventilated Group (VM). The variables analyzed were respiratory mechanics and hemodynamics parameters: heart frequency, breath frequency, systolic arterial pressure, diastolic arterial pressure, peripheral oxygen saturation, peak inspiratory pressure, plateu lung pressure, dynamic lung compliance and static lung compliance. Data were collected happened at four moments: before procedure, immediately after procedure, 5 minutes and 30 minutes before procedure. Results: The results showed respiratory mechanic and hemodynamics repercussions in the three groups analyzed. When comparing the three groups, was observed significant alteration in variables breath frequency at the period 30 minutes after procedure between RM and VM groups $(p=0,007)$, peak inspiratory pressure at the period 30 minutes after procedure between $\mathrm{RM}$ and FiO2 groups $(\mathrm{p}=0,005)$ and dynamic lung compliance at the period 30 minutes after procedure between FiO2 and VM groups $(\mathrm{p}=0,005)$. Conclusion: In conclusion, the three suction complementary methods presented some respiratory mechanic and hemodynamics repercussions, but all of them can be considerate applicable.
\end{abstract}

Keywords: Suction. Respiratory Mechanics. Hemodynamics.

Correspondência: Guilherme Pinheiro Ferreira da Silva, Av. Rogaciano Leite, 200, apt 403C. Salinas - Fortaleza/Ceará, Fone: (85) $3241-4273$. E-mail: guilhermepinheiro87@hotmail.com

Conflito de interesses: não há conflito de interesses.

Recebido em 11 Out 2013; Revisado em 14 Nov 2013; Aceito em 29 Nov 2013. 


\section{INTRODUÇÃO}

A aspiração traqueobrônquica (ATB) é um procedimento invasivo, desconfortável e de alto risco; portanto deve ser realizado somente quando necessário. A técnica está indicada nas seguintes situações: secreção visível na luz do tubo; alterações na ausculta pulmonar e radiológicas consistentes com acúmulo de secreção; aumento aparente do trabalho respiratório; hipoxemia, hipercapnia ou queda na saturação de oxigênio; alterações gráficas de fluxo ou pressão; suspeita de aspiração gástrica; aumento da pressão de pico inspiratório durante a ventilação artificial com volume controlado ou redução do volume corrente durante a ventilação artificial com pressão controlada ${ }^{1-3}$.

A remoção de secreção das vias aéreas por meio da ATB auxilia na higiene brônquica, na troca gasosa, na redução do trabalho respiratório e na melhora da mecânica ventilatória. $\mathrm{O}$ estudo de Moreira e cols ${ }^{4}$ evidenciou que após a realização de um protocolo de higiene brônquica, houve uma diminuição do shunt intrapulmonar, aumento da complacência total e melhora dos gases arteriais em até duas horas após o fim do protocolo. Quanto às repercussões hemodinâmicas, Rodrigues e cols5 demonstraram que houve aumento da frequência cardíaca imediatamente, 3 e 10 minutos após a realização de protocolos de remoção de secreção e da pressão arterial média 8 minutos após a aspiração em pacientes secretivos.

O benefício da ATB em manter as vias aéreas pérvias é bem definido, porém podem ocorrer intercorrências como: a hipoxemia gerada pela interrupção da ventilação mecânica, pela perda de PEEP e da fração inspirada de oxigênio ( $\mathrm{FiO}_{2}$ ) desejada ${ }^{(1,4,5)}$.

A hipoxemia é comumente definida como uma baixa significante na pressão arterial de oxigênio $\left(\mathrm{PaO}_{2}\right)$, ficando abaixo dos níveis basais (que variam de 80 a $100 \mathrm{mmHg}$ ) ${ }^{6,7}$.

A insuficiência respiratória é outra responsável por quadros hipoxêmicos. Trata-se de um distúrbio provocado por qualquer condição que afete a capacidade dos pulmões em realizar as trocas gasosas ou eliminar dióxido de carbono, por exemplo, acúmulo de secreção que irá interferir diretamente na relação ventilação-perfusão $(\mathrm{V} / \mathrm{Q})^{8}$.

Em consequência à hipoxemia, o paciente pode apresentar arritmias, parada cardíaca e morte, além de lesões no sistema nervoso central. Como estratégias para a redução desses efeitos, podem ser utilizadas a hiperventilação através do ressuscitador manual (RM) ou do ventilador mecânico (VM) ou a hiperoxigenação, por meio do aumento da $\mathrm{FiO}_{2}{ }^{1,6,7}$.

A hiperventilação manual (HM) gera uma pressão positiva inspiratória para o paciente por meio de um RM ou através do VM, além de ofertar um volume corrente (VC) superior ao que normalmente o ventilador oferece ao paciente e é capaz de aumentar a capacidade residual funcional (CRF) $)^{1,9-12}$.
A HM utilizando RM consiste na ventilação pulmonar utilizando uma bolsa de hiperventilação manual, com o objetivo de prevenir e/ou reexpandir áreas colapsadas, por meio dos canais colaterais, distribuir uniformemente o gás, melhorando a oxigenação sanguínea e a complacência do pulmão, além de deslocar secreções de regiões periféricas para regiões mais centrais a fim de serem aspiradas ${ }^{7,9,12-16}$.

Outra forma de prevenir a hipoxemia induzida pela ATB é através da hiperoxigenação prévia ao procedimento, obtida com a elevação da $\mathrm{FiO}_{2}$ a $100 \%$ por um tempo maior que um minuto, assegurando uma saturação de oxigênio $\left(\mathrm{SpO}_{2}\right)$ adequada, prevenindo repercussões como: taquicardia, arritmia, aumento da frequência respiratória, hipertensão ou hipotensão ${ }^{1,8,11,17}$.

Dentro desse contexto, o presente estudo justifica-se na identificação do método complementar à ATB mais adequado para prevenir a hipoxemia, menores repercussões hemodinâmicas e da mecânica respiratória associada aos procedimentos fisioterapêuticos; portanto, poderá proporcionar menores danos aos pacientes submetidos aos procedimentos de remoção de secreção, culminando em uma maior segurança ao profissional durante a realização destas técnicas. Dessa forma, este estudo tem como objetivo analisar os efeitos de três métodos complementares à ATB nas repercussões hemodinâmicas e mecânica respiratória em pacientes sob ventilação mecânica invasiva.

\section{MÉTODOS}

Foi realizada uma pesquisa de caráter intervencionista, simples cego e com abordagem quantitativa no período de janeiro a maio de 2013. Esta pesquisa foi aprovada pelo Comitê de Ética e Pesquisa da Universidade de Fortaleza (parecer número 272.982) e seguiu os preceitos éticos segundo a resolução 196/96 do Conselho Nacional de Saúde que estabeleceu os princípios para pesquisa em seres humanos ${ }^{18}$.

Participaram do estudo 45 pacientes com diagnóstico clínico de insuficiência respiratória, independente da patologia de base, internados na Unidade de Terapia Intensiva (UTI) de um hospital privado do município de Fortaleza/CE e que estavam em uso de ventilação mecânica invasiva, independente do gênero, com estabilidade hemodinâmica, cuja família e/ ou responsável concordou com a participação do mesmo mediante assinatura do termo de consentimento livre e esclarecido. Todos os pacientes estavam em decúbito dorsal e com cabeceira elevada à 45 ㅇ.

Foram excluídos do estudo os pacientes com história de fratura de costela, presença de dreno torácico, broncoespasmo grave, pneumotórax, uso de suporte ventilatório com altos níveis de pressão positiva expiratória final (acima de $12 \mathrm{cmH2O}$ ) e sistema de aspiração fechado. 
Os pacientes foram divididos de forma aleatória, por meio de sorteio, em três grupos compostos por 15 indivíduos cada: Grupo Ressuscitador Manual (RM) da marca $\mathrm{AMBU}^{\circledR}$, Grupo Fração inspirada de Oxigênio $\left(\mathrm{FiO}_{2}\right)$ e grupo Ventilação Mecânica (VM).

Os pacientes foram submetidos a três métodos complementares à ATB o grupo RM, foram realizadas 6 ventilações com auxílio do ressuscitador manual. No grupo $\mathrm{FiO}_{2}$, a $\mathrm{FiO}_{2}$ do paciente foi mantida a $100 \%$ durante dois minutos antes e após a realização da técnica de aspiração. No grupo VM, os pacientes foram ventilados manualmente 6 vezes a partir do uso da tecla manual cycle do ventilador mecânico antes do procedimento de ATB. Estes procedimentos foram baseados em alguns critérios da American Association for Respiratory Care que rege os procedimentos de aspiração traqueal (19).

A ATB foi realizada de forma asséptica e rápida, com instilação de $2-10 \mathrm{ml}$ de solução salina 0,9\% para fluidificar a secreção. A técnica consistiu em três a cinco introduções e cada introdução teve o tempo de 12 segundos ${ }^{1,4}$.

As variáveis analisadas consistiram nos parâmetros da mecânica respiratória e cardiorrespiratórios: frequência cardíaca (FC), frequência respiratória (FR), pressão arterial sistólica (PAS), pressão arterial diastólica (PAD), saturação periférica de oxigênio $\left(\mathrm{SpO}_{2}\right)$, pressão de pico (P. Pico), pressão de platô (P. Platô), complacência estática (C. Estática), complacência dinâmica (C. Dinâmica).

A coleta de dados ocorreu em quatro momentos distintos: antes da realização do procedimento, imediatamente após, 5 minutos e 30 minutos após o procedimento.

Os protocolos e a coleta dos dados foram realizados apenas pelas pesquisadoras.

O tamanho amostral foi calculado para obter um padrão de $90 \%$ com $p<0,05$ na variável saturação periférica de oxigênio.

Os dados obtidos foram analisados estatisticamente por um pesquisador que não participou diretamente da coleta de dados, por meio do programa SPSS, versão 13.0. Para verificar a homogeneidade da amostra foi utilizado o teste de Kolmogorov-Smirnov e o teste ANOVA para medidas repetidas, sendo considerado como estatisticamente significantes os valores de $p<0,05$.

\section{RESULTADOS}

Em relação às características clínicas basais dos grupos estudados, não houve diferença estatística significante na comparação das variáveis: idade, gênero, FC, FR, PAS, $\mathrm{PAD}, \mathrm{SpO}_{2}, \mathrm{P}$. Pico, P. Platô e C. Dinâmica, demonstrando homogeneidade da amostra $(p>0,05)$. No entanto, a variável $C$. Estática apresentou diferença significante entre os grupos estudados $(p<0,05)$ (Tabela 1$)$.
Tabela 1 Características clínicas basais dos 45 pacientes do estudo, divididos em 3 grupos: Grupo RM ( $n=15)$, Grupo $\mathrm{FiO}_{2}(n=15)$ e Grupo VM ( $\left.n=15\right)$.

\begin{tabular}{ccccc}
\hline Variável & $\begin{array}{c}\text { Grupo RM } \\
(\mathrm{n}=15)\end{array}$ & $\begin{array}{c}\text { Grupo Fi02 } \\
(\mathrm{n}=15)\end{array}$ & $\begin{array}{c}\text { Grupo VM } \\
(\mathrm{n}=15)\end{array}$ & $\mathrm{p}$ \\
\hline Idade (anos) & $64,4 \pm 18,5$ & $63,9 \pm 19,6$ & $72,2 \pm 20,9$ & $\mathrm{~ns}$ \\
Gênero (M/F) & $8 / 7$ & $8 / 7$ & $4 / 11$ & - \\
FC (bpm) & $86,7 \pm 20,2$ & $85 \pm 15,7$ & $83,8 \pm 23,2$ & $\mathrm{~ns}$ \\
FR (rpm) & $16,2 \pm 7,8$ & $17 \pm 4,9$ & $20,3 \pm 8,6$ & $\mathrm{~ns}$ \\
PAS (mm/Hg) & $139,2 \pm 33,8$ & $128,7 \pm 27$ & $128,4 \pm 29,9$ & $\mathrm{~ns}$ \\
PAD (mm/Hg) & $81 \pm 22,9$ & $71 \pm 17,5$ & $69,2 \pm 13,5$ & $\mathrm{~ns}$ \\
Sp02 (\%) & $95 \pm 4,5$ & $96,5 \pm 3,6$ & $96 \pm 4$ & $\mathrm{~ns}$ \\
P. Pico & $27,4 \pm 8,2$ & $29,6 \pm 7,6$ & $28,5 \pm 7,4$ & $\mathrm{~ns}$ \\
P. Platô & $21,3 \pm 6,5$ & $23,2 \pm 5,7$ & $26 \pm 11$ & $\mathrm{~ns}$ \\
C. Estática & $35,5 \pm 16,3$ & $31,8 \pm 8,7$ & $52,8 \pm 33,6 *$ & \\
C. Dinâmica & $25,3 \pm 10,3$ & $23,3 \pm 7,8$ & $28 \pm 24$ & $\mathrm{~ns}$ \\
\hline
\end{tabular}

$\mathrm{FiO}_{2}=$ Fração inspirada de oxigênio; $\mathrm{VM}=$ Ventilação mecânica; $\mathrm{M}=$ Masculino; F= Feminino; FC= Frequência cardíaca; FR= Frequência respiratória; $\mathrm{PAS}=$ Pressão arterial sistólica; $\mathrm{PAD}=$ Pressão arterial diastólica; $\mathrm{SpO}_{2}=$ Saturação de Oxigênio; P.Pico= Pressão de pico; P.Platô= Pressão de platô; C. Estática= Complacência estática; C. Dinâmica $=$ Complacência dinâmica; $b p m=$ batimentos por minuto; rpm= respirações por minuto; $\mathrm{mm} / \mathrm{Hg}=$ milímetros de Mercúrio; \%= percentual. ns= Não significante.

* Grupo FiO2 versus Grupo VM.

Quanto aos parâmetros cardiorrespiratórios, no grupo RM houve diferença significativa somente na variável FC no período imediatamente após a intervenção quando comparado com o período 30 minutos pós-intervenção $(p=0,001)$. O grupo $\mathrm{FiO}_{2}$, apresentou diferença significativa nas variáveis $\mathrm{FC}$ e $\mathrm{SpO}_{2}$ no período pré-intervenção versus imediatamente após $(p=0,04$, $p=0,01$, respectivamente). No grupo VM, houve diferença significativa na variável $\mathrm{FC}$ nos períodos pré-intervenção versus imediatamente após, pós versus 5 minutos pós-intervenção e pós versus 30 minutos pós-intervenção $(p<0,05)$ (Tabela 2).

Em relação aos parâmetros da mecânica respiratória, houve diferença significativa apenas nas variáveis P. Pico e C. Dinâmica no período pré versus 30 minutos pósintervenção no grupo RM ( $p=0,04)$ (Tabela 3).

Quando comparado às variáveis hemodinâmicas entre os grupos, encontrou-se diferença significativa apenas na variável FR no período 30 minutos pós-intervenção do grupo RM versus o grupo VM ( $p=0,007)$ (Tabela 2). Enquanto que, nas variáveis da mecânica respiratória, encontrou-se diferença significativa entre os grupos $\mathrm{RM}$ e $\mathrm{FiO}_{2}$ na variável P. Pico no período 30 minutos pós-intervenção $(p=0,005)$ e entre os grupos FiO2 e VM na variável C. Dinâmica no período 30 minutos pós-intervenção $(p=0,005)$ (Tabela 3). 
Tabela 2 Parâmetros cardiorrespiratórios nos quatro períodos de coleta do Grupo RM, do Grupo $\mathrm{FiO} 2$ e do Grupo VM.

\begin{tabular}{lllll}
\hline Variável & $\begin{array}{l}\text { Grupo RM } \\
(n=15)\end{array}$ & $\begin{array}{l}\text { Grupo Fi02 } \\
(n=15)\end{array}$ & $\begin{array}{l}\text { Grupo VM } \\
(n=15)\end{array}$ & $p$ \\
\hline
\end{tabular}

FC (bpm)

Pré

Pós

5' - Pós

30 ‘ Pós

$P$

$\mathrm{FR}(\mathrm{rpm})$

Pré

Pós

5'-Pós

30 '- Pós

$\mathrm{P}$

PAS $(\mathrm{mm} / \mathrm{Hg})$

Pré

Pós

5' - Pós

30 '- Pós

$P$

$\mathrm{PAD}(\mathrm{mm} / \mathrm{Hg})$

Pré

Pós

5'-Pós

30 ‘- Pós

P

Sp02 (\%)

Pré

Pós

5' - Pós

30 ‘ - Pós

$86,7 \pm 20,2 \quad 85 \pm 15,7 \square \quad 83,8 \pm 23,2 \square \quad n s$

$98,6 \pm 28,9 \quad 92,2 \pm 16,6$

$90,2 \pm 22,2 €$ ns

$92,6 \pm 26,9 \quad 87,4 \pm 17,4$

$85,5 \pm 23,6 \quad n s$

$86,4 \pm 22,4^{\dagger} \quad 90,3 \pm 18,8$

$82,3 \pm 19,7+$ ns

0,001

0,04

$<0,05$

$20,3 \pm 8,6 \quad$ ns

$16,2 \pm 7,8 \quad 17 \pm 4,9$

$20,6 \pm 8,5 \quad$ ns

$16,1 \pm 5,4 \quad 17,5 \pm 5,5$

$19,2 \pm 7,1 \quad$ ns

$15,3 \pm 5,5 \quad 15,9 \pm 4,2$

$19,2 \pm 6,4 \# \quad 0,007$

Ns

$15,8 \pm 4,5$

ns

ns

$139,2 \pm 33,8 \quad 128,7 \pm 27 \quad 128,4 \pm 29,9 \quad$ ns

$136,8 \pm 36,2 \quad 132,2 \pm 28,6 \quad 128,4 \pm 27,1 \quad$ ns

$131,8 \pm 29,6 \quad 122,2 \pm 27,7 \quad 126,4 \pm 24,4 \quad n s$

$124 \pm 26,2 \quad 131 \pm 23,5 \quad 132,7 \pm 28,4 \quad$ ns

Ns

ns

ns

$\mathrm{P}$ $81 \pm 22,9$

$71 \pm 17,5$

$69,2 \pm 13,5 \quad$ ns

$84 \pm 27,8 \quad 72,6 \pm 18,7$

$70,9 \pm 12,4 \quad$ ns

$80,7 \pm 20,6$

$73,8 \pm 22,3$

$68,6 \pm 12,3 \quad n s$

$73,4 \pm 20,2$

$72,5 \pm 17$

$70,2 \pm 12,9 \quad$ ns

Ns

ns

ns

$\mathrm{FiO}_{2}=$ Fração inspirada de oxigênio; $\mathrm{VM}=$ Ventilação mecânica; $M=$ Masculino; $F=$ Feminino; $F C=$ Frequência cardíaca; $F R=$ Frequência respiratória; $P A S=$ Pressão arterial sistólica; $\mathrm{PAD}=$ Pressão arterial diastólica; $\mathrm{SpO}_{2}=$ Saturação de Oxigênio; bpm= batimentos por minuto; $r p m=$ respirações por minuto; $\mathrm{mm} / \mathrm{Hg}=$ milímetros de Mercúrio; $\%=$ percentual. ns= Não significante.

\# Grupo RM versus Grupo VM; € Pós versus 5' pósintervenção

† Pós versus 30’ pós-intervenção; $\square$ Pré versus pósintervenção;
Tabela 3 Parâmetros da mecânica respiratória nos quatro períodos de coleta do Grupo RM, do Grupo FiO2 e do Grupo VM.

\begin{tabular}{|c|c|c|c|c|}
\hline Variável & $\begin{array}{c}\text { Grupo RM } \\
(\mathrm{n}=15)\end{array}$ & $\begin{array}{c}\text { Grupo Fi02 } \\
(n=15)\end{array}$ & $\begin{array}{c}\text { Grupo VM } \\
(\mathrm{n}=15)\end{array}$ & $P$ \\
\hline \multicolumn{5}{|l|}{ P. Pico } \\
\hline Pré & $27,4 \pm 8,2+$ & $29,6 \pm 7,6$ & $28,5 \pm 7,4$ & \\
\hline Pós & $25,2 \pm 6,2$ & $31,5 \pm 11$ & $27,1 \pm 7,2$ & \\
\hline 5' - Pós & $23,9 \pm 6,5$ & $30,3 \pm 11,1$ & $27,8 \pm 8$ & \\
\hline 30 ‘-Pós & $21,2 \pm 6,8^{\#}$ & $30,8 \pm 10,9$ & $28,4 \pm 7,7$ & 0,005 \\
\hline$P$ & 0,04 & ns & ns & \\
\hline \multicolumn{5}{|l|}{ P. Platô } \\
\hline Pré & $21,3 \pm 6,5$ & $23,2 \pm 5,7$ & $26 \pm 11$ & \\
\hline Pós & $22,5 \pm 7,1$ & $23 \pm 6,5$ & $24,6 \pm 8,3$ & \\
\hline 5' - Pós & $20,6 \pm 3,8$ & $23,6 \pm 8$ & $23,8 \pm 9,2$ & \\
\hline 30 ‘-Pós & $21,3 \pm 5,2$ & $23,2 \pm 6,3$ & $23,4 \pm 9$ & \\
\hline$P$ & ns & $\mathrm{ns}$ & ns & \\
\hline \multicolumn{5}{|l|}{ C. Estática } \\
\hline Pré & $35,5 \pm 16,3$ & $31,8 \pm 8,7$ & $52,8 \pm 33,6$ & 0,04 \\
\hline Pós & $31,9 \pm 13,4$ & $34 \pm 13,7$ & $37,6 \pm 33,1$ & \\
\hline 5' - Pós & $35,3 \pm 14,2$ & $34,1 \pm 12,7$ & $51,4 \pm 44,6$ & \\
\hline 30 ‘-Pós & $45,5 \pm 43,6$ & $31,7 \pm 8$ & $42,2 \pm 35,1$ & \\
\hline $\mathrm{P}$ & ns & ns & ns & \\
\hline \multicolumn{5}{|l|}{ C. Dinâmica } \\
\hline Pré & $25,3 \pm 10,3+$ & $23,3 \pm 7,8$ & $28 \pm 24$ & \\
\hline Pós & $27,6 \pm 9,9$ & $22,2 \pm 9,1$ & $27,9 \pm 13,8$ & \\
\hline 5' - Pós & $28,1 \pm 9,1$ & $24 \pm 9,3$ & $26,1 \pm 10,6$ & \\
\hline 30 ‘- Pós & $35,1 \pm 12$ & $23,2 \pm 9 *$ & $27 \pm 16,1$ & 0,005 \\
\hline$P$ & 0,04 & ns & ns & \\
\hline
\end{tabular}

P.Pico= Pressão de pico; P.Platô $=$ Pressão de platô;

C. Estática = Complacência estática; C. Dinâmica= Complacência dinâmica.

* Grupo Fi02 versus Grupo VM

\# Grupo RM versus Grupo FiO2

† Pré versus 30' pós-intervenção 


\section{DISCUSSÃO}

O presente estudo verificou que houve repercussões na hemodinâmica e na mecânica respiratória nos três grupos analisados.

Diniz e colaboradores ${ }^{20}$ elaboraram um protocolo de aspiração em que foram analisadas as repercussões hemodinâmicas relacionadas a duas formas de hiperoxigenação: $\mathrm{FiO}_{2}$ a $100 \%$ e a $50 \%$ dos níveis basais, sendo observado aumento da FC no 1 - minuto após a realização da técnica no grupo com $\mathrm{FiO}_{2} 50 \%$ acima do valor inicial, fato que não ocorreu na hiperoxigenação com FiO2 a 100\%. Evidenciou-se, também, o aumento da $\mathrm{SpO}_{2}$ e FR mais significante no grupo com FiO2 à $100 \%$ no primeiro minuto após a aspiração quando comparado aos níveis basais. Tal fato corrobora o presente estudo, em que se verificou que o grupo $\mathrm{FiO}_{2}$ aumentou a $\mathrm{FC}$ e a $\mathrm{SpO}_{2}$ imediatamente após a ATB.

Na análise intragrupo, a variável FR apresentou redução nos três grupos estudados, porém sem significância estatística. Este achado não corrobora o estudo anterior(21) em que foi encontrado aumento da FR no momento imediatamente após a aspiração com retorno aos níveis basais após 30 minutos de realização da técnica.

Este estudo evidenciou diminuição da P. Pico e aumento $C$. Dinâmica quando comparados os períodos pré e 30 minutos pós-intervenção no grupo RM. Em contrapartida, um estudo em que se comparou um protocolo se fisioterapia composto por: compressão torácica manual por 10 minutos, hiperinsuflação manual com $\mathrm{O}_{2}$ a $10 \mathrm{~L} / \mathrm{min}$, instilação de 5 a $10 \mathrm{~mL}$ de solução fisiológica a 0,9\%, aspiração traqueal e hiperinsuflação manual com $\mathrm{O}_{2}$ a $10 \mathrm{~L} / \mathrm{min}$ (12 rpm) por um minuto versus somente aspiração, evidenciou-se que não houve alteração significativa da C. Dinâmica nos momentos: imediatamente após, 30 minutos e 120 minutos após a realização do protocolo, tanto na análise intragrupo quanto na análise intergrupo ${ }^{21}$.

As variáveis P. Platô e C. Estática não apresentaram alteração estatisticamente relevante, corroborando com dois estudos anteriores em que ambos comparavam aspiração isolada, técnica de Bag-Squeezing e PEEPZEEP e encontraram resultados semelhantes ao presente trabalho4,5. Adicionalmente, outra pesquisa em que se comparava ATB isolada à ATB associada à HM através do RM e da compressão torácica, não evidenciou alteração significativa na P. Platô e C. Estática ${ }^{13}$.

\section{CONCLUSÃO}

Conclui-se que houve repercussões hemodinâmicas e de mecânica respiratória em todos os três métodos complementares a aspiração, porém todos são considerados seguros e aplicáveis. O presente estudo apresenta algumas limitações como a não verificação da quantidade de secreção aspirada, modo de ventilação mecânica utilizada e comparação entre os diferentes tipos de patologia. Dessa forma, é preciso a ampliação dos experimentos para apontar de forma definitiva a repercussão dos métodos complementares apresentados.

\section{REFERÊNCIAS}

1. Araújo SG, Machado MGR. Aspiração endotraqueal. In: Machado MGR. Bases da fisioterapia respiratória: terapia intensiva e reabilitação. Rio de Janeiro: Guanabara Koogan; 2008. p. 66-77.

2. Pagotto IM, Oliveira LRC, Araújo FCLC, Carvalho NA, Chiavone P. Comparação entre os sistemas aberto e fechado de aspiração: revisão sistemática. Rev Bras Ter Intensiva [Internet]. 2008 Dez [acesso 2013 Jul 11]; 20(4):331-338. Disponível em: http://www.scielo.br/scielo. php?script=sci_arttext\&pid=S0103-507X2008000400003\&Ing=en. doi: http://dx.doi.org/10.1590/S0103-507X2008000400003.

3. Martins JJ, Maestri E, Dogenski D, Nascimento RP, Silva RM, Gama FO. Necessidade de aspiração de secreção endotraqueal: critérios utilizados por uma equipe de enfermagem de uma unidade de terapia intensiva. Cienc Cuid Saúde [Internet]. 2008 [acesso 2013 Jul 11]; 7(4):517-522. Disponível em: http://periodicos.uem.br/ojs/index. php/CiencCuidSaude/article/view/6660/3918. doi: http://dx.doi.org/ 10.4025/cienccuidsaude.v7i4.6660.

4. Moreira FC. Comportamento da mecânica ventilatória durante a realização de um atendimento de fisioterapia respiratória [dissertação de mestrado] [Internet]. Porto Alegre: Universidade Federal do Rio Grande do Sul; 2009 [acesso 2013 Jul 11]. Disponível em: http://hdl. handle.net/10183/26902.
5. Rodrigues MVH. Estudo do comportamento hemodinâmico, da troca gasosa, da mecânica respiratória e da análise do muco brôquico na aplicação de técnicas de remoção de secreção brônquica em pacientes sob ventilação mecânica [tese de doutorado] [Internet]. São Paulo: Universidade de São Paulo; 2007 [acesso 2013 Jul 11]. Disponível em: http://www.teses.usp.br/teses/disponiveis/5/5131/tde-10032008132615/pt-br.php.

6. Abreu LC, Pereira VX, Valenti VE, Panzarin SA, Moura Filho OF. Uma visão da prática de fisioterapia respiratória: ausência de evidência não é evidência de ausência. Arq Med Abc [Internet]. 2007 [acesso 2013 Jul 11];32(2):76-8. Disponível em: http://www.fisioterapia.com/public/ files/artigo/artigo06_1.pdf.

7. Cerqueira Neto ML. Avaliação da segurança das manobras de fisioterapia respiratória de vibrocompressão, aumento de fluxo expiratório e aspiração em pacientes críticos com traumatismo cranioencefálico [tese de doutorado] [Internet]. Curitiba: Universidade Federal do Paraná; 2012 [acesso 2013 Jul 11]. Disponível em: http:// hdl.handle.net/1884/29371.

8. Presto B, Damázio L. Fisioterapia Respiratória. 4. ed. Rio de Janeiro: Elsevier; 2009. 
9. Lemes DA, Guimarães FS. O Uso da Hiperinsuflação como Recurso Fisioterapêutico em Unidade de Terapia Intensiva. Rev Bras de Ter Intensiva [Internet]. 2007 [acesso 2013 Jul 11]; 19(2):222-25. Disponível em: http://www.scielo.br/scielo.php?script=sci_arttext\&pid=S0103507X2007000200014\&lng=en. doi: http://dx.doi.org/10.1590/S0103$507 \times 2007000200014$.

10. Ortiz TA. Influência do operador e do reanimador manual na manobra de hiperinsuflação manual: estudo em simulador do sistema respiratório [dissertação de mestrado] [Internet]. São Paulo: Universidade de São Paulo; 2008. Disponível em: http://www.teses. usp.br/teses/disponiveis/5/5160/tde-25032009-091656/publico/ tatianaaortiz.pdf.

11. Sarmento GJV, Vega JM, Lopes NS. Fisioterapia em UTI. São Paulo: Atheneu; 2010.

12. Lobo DML, Cavalcante LA, Mont'Alverne DGB. Aplicabilidade das técnicas de bag squeezing e manobra zeep em pacientes submetidos à ventilação mecânica. Rev Bras Ter Intensiva [Internet]. 2010 [acesso 2013 Jul 11]; 22(2):186-91. Disponível em: http://www.scielo.br/scielo.php?script=sci_arttext\&pid=S0103507X2010000200013\&lng=en. doi: http://dx.doi.org/10.1590/ S0103-507X2010000200013.

13. Dias $C M$, Sirqueira TM, Faccio TR, et al. Efetividade e segurança da técnica de higiene brônquica: hiperinsuflação manual com compressão torácica. Rev Bras Ter Intensiva [Internet]. 2011 [acesso 2013 Jul 11]; 23(2):190-198. Disponível em: http://www.scielo.br/scielo. php?script=sci_arttext\&pid=S0103-507X2011000200012\&Ing=en. doi: http://dx.doi.org/10.1590/S0103-507X2011000200012.

14. Godoy ACF, Yokota CO, Araújo IIM, Freitas MIP. Manobras de Hiperinflação Manual Podem Causar Aspiração de Secreções Orofaríngeas em Paciente sob Ventilação Mecânica? Rev Bras Anestesiol [Internet]. 2011 [acesso 2013 Jul 11]; 61(5):556-560. Disponível em: http://www.scielo.br/scielo.php?script=sci_ arttext\&pid=S0034-70942011000500005\&Ing=en. doi: http://dx.doi. org/10.1590/s0034-70942011000500005.

15. Jelic S, Cunningham JA, Factor P. Clinical review: airway hygiene in the intensive care unit. Crit Care [Internet]. 2008 [cited 2013 Jul 11];12(2):209. Available from: http://www.ncbi.nlm.nih.gov/ pmc/articles/PMC2447567/pdf/cc6830.pdf. doi: http://dx.doi. org/10.1186/cc6830. Epub 2008 Mar 31. Review. PubMed PMID: 18423061; PubMed Central PMCID: PMC2447567.
16. Godoy ACF, Vieira RJ. Comparação da FiO2 fornecida por sete modelos de sistema balão-máscara auto-inflável. Rev Bras Anestesiol. 2009; 59(1): 21-27.

17. Jerre G, Silva TJ, Beraldo MA, Gastaldi A, Kondo C, Leme F, et al. Fisioterapia no paciente sob ventilação mecânica. J Bras Pneumol [Internet]. 2007 [acesso 2013 Jul 11]; 33 (Supl 2):S142-50. Disponível em: http://www.scielo.br/scielo.php?script=sci_arttext\&pid=S180637132007000800010\&Ing=en. doi: http://dx.doi.org/10.1590/ S1806-37132007000800010.

18. Ministério da Saúde (BR). Resolução CNS no 196, de 10 de outubro de 1996. Aprova diretrizes e normas regula-mentadoras de pesquisa envolvendo seres humanos. Diário Oficial da União, Brasília, 1996;201:21082.

19. American Association for Respiratory Care. AACR Clinical Practice Guidelines. Endotracheal suctioning of mechanically ventilated patients with artificial airways 2010. Respir Care [Internet]. 2010 Jun [cited 2013 Jul 11];55(6):758-64. Available from: http://rc.rcjournal. com/content/55/6/758.full.pdf+html. PubMed PMID: 20507660.

20. Diniz, GCLM, Souza AO, Oliveira HMD, Arrais RCS, Pessoa BP. Figueiredo PHS. A utilização de $\mathrm{FiO2}$ inferior a $100 \%$ para hiperoxigenação de pacientes estáveis submetidos à aspiração endotraqueal. ASSOBRAFIR Cienc [Internet]. 2012 [acesso $2013 \mathrm{Jul}$ 11]; 3(1):45-56. Disponível em: http://www.uel.br/revistas/uel/ index.php/rebrafis/article/view/10886/10598.

21. Rosa FK, Roese CA, Savi A, Dias AS, Monteiro MB. Comportamento da mecânica pulmonar após a aplicação de protocolo de fisioterapia respiratória e aspiração traqueal em pacientes com ventilação mecânica invasiva. Rev Bras Ter Intensiva [Internet]. 2007 [acesso 2013 Jul 11];19(2):170-5. Disponível em: http://www.scielo.br/scielo. php?script=sci_arttext\&pid=S0103-507X2007000200005\&Ing=en. doi: http://dx.doi.org/10.1590/\$0103-507X2007000200005.

Como citar este artigo / How to cite this article:

Bezerra C, Águila DX, Araújo FCS, da Silva GPF. Efeitos de três métodos complementares à aspiração nas repercussões hemodinâmicas e mecânica respiratória em pacientes sob ventilação mecânica invasiva. J Health Biol Sci. 2013 Out-Dez; 1(4):154-159. 